International Journal of Biology, Pharmacy and Allied Sciences (IJBPAS) 'A B Bridgs Between Caboratory and QRader'

WwW.iibpas.com

PREVALENCE OF ANTIBIOTIC RESISTANCE AND BIOFILM PRODUCING BACTERIAL ISOLATES ON FOODS OF ANIMAL ORIGIN AND FRESH FRUIT JUICES

\title{
AMUTHA $T^{1}$ AND RAJESWARI $\mathbf{S}^{2 *}$
}

1: Ph.D, Research scholar, Department of Botany, Bharathiar University, Coimbatore, TN, India

2: Associate professor, Department of Botany, P.S.G. College of Arts and Science,

$$
\text { Coimbatore, TN, India }
$$

*Corresponding Author: Rajeswari S: E Mail: psgbotany1978@gmail.com; +919486312811

Received $30^{\text {th }}$ March 2020; Revised $25^{\text {th }}$ April 2020; Accepted $15^{\text {th }}$ July 2020; Available online $1^{\text {st }} \mathrm{Jan} .2021$

\section{https://doi.org/10.31032/IJBPAS/2021/10.1.5329}

\section{ABSTRACT}

Antimicrobial resistance is a major global problem for both humans and animals. Increased use of antimicrobials in livestock and poultry was one of the causes of the development of antimicrobial resistance in microorganisms. The aim of the study was to analysis the antimicrobial resistant bacteria from raw meat and fruit juice samples. A total of 7 bacterial genera were recovered from meat and fruit samples. Out of 24 samples, 48 isolates were observed and meat showed the highest number of isolates. E.coli, K.pneumoniae and E.faecalis $(21 \%)$ were obtained to be the most prevalent bacteria followed by Proteus $\mathrm{sp}$ (12.5\%). Following the prevalence study, antibacterial resistance patterns were observed from all isolates. From the overall results in both sources, among the 11 antibiotics used, penicillin and amoxicillin were resistant to $93.3 \%$ of isolates, and a higher number of MDR bacteria were observed in goat samples in comparison to other samples. Additionally, $60.4 \%$ of biofilm producers were observed from all samples, especially those which were higher in MDR isolates. This study indicates that antibiotics are at high risk of bacteria, higher in meat than in fruit juices.

Keywords: Meat, fruit juice, MDR, Biofilm, food pathogens 


\section{INTRODUCTION}

Foodborne diseases are caused by ingestion of contaminated food or drink. Infinite microbes and toxic substances can contaminate foods. There are more than 250 known foodborne diseases all over the world. The majority of infections were caused by bacteria, viruses, and parasites. Other foodborne diseases are essentially poisonings caused by toxins, chemicals contaminating the food. All foodborne microbes and toxins pass through the gastrointestinal tract into the body and often cause the first symptoms, Nausea, vomiting, abdominal cramps and diarrhea are frequent in foodborne diseases.

Foodborne diseases are occurring at any point in the food production, supply and consumption chain. This illness was caused by environmental factors like water, soil, or air pollution, and improper storage and processing of foods. Many microbes can spread in more than one way, so it may not be immediately apparent that a foodborne illness [1].

The foodborne infection was occurred through contaminated raw fruits and vegetables, raw and improperly cooked eggs, poultry and meat, raw milk and milky products that are made with unpasteurized milk. It also can be transmitted through contact with infected animals or infected food handlers who have not hygienic [2].
Food-producing animals (e.g., cattle, chickens, pigs, and turkeys) are the major reservoirs for many foodborne pathogens such as Campylobacter species, non-Typhi serotypes of Salmonella enterica, Shiga toxin-producing strains of Escherichia coli, and Listeria monocytogenes [3]. A number of researches have stated the outbreak of infections due to the intake of infected meat and meat products. Karaoke et al [4] report that 1.5 billion diarrhea and 3 million deaths worldwide are caused by foodborne infections.

A recent study in India found that bacteria resistant to most potent antibiotics, which were present in fresh food samples including fruits, chicken and fish. This incidence of antimicrobial-resistant is associated with the usage of antimicrobial agents in food producing animals. Food of animal origin carrying a variety of pathogenic and non-pathogenic bacteria has become a vector for interactions which could lead to the creation of new drugresistant and multidrug-resistant (MDR) bacteria through horizontal exchange of drug-resistant genes, which isolates were not easily eradicated and isolates causing diseases are not easily cured [5 \& 6].

Several researchers have reported variable prevalence rates of bacterial infection in different parts of India. The 
common antimicrobial-resistant bacteria including Escherichia coli, Klebsiella pneumoniae, Staphylococcus aureus, Streptococcus pneumoniae, and Salmonella spp., which are the common human normal microbiota as well as important human pathogens, have been reported to be the main forum among foodborne pathogens [7]. In developed and developing countries, the risk of antimicrobial-resistant bacteria affects the economies and health of people.

Both developing countries and developed countries have implemented various national surveillance programs for control of antimicrobial resistance microbes. Nevertheless, some countries completely lack data regarding resistance to antimicrobial agents. Hence the goal of this study was to determine the antibiotic resistance pattern of bacterial isolates from different types of meat and fruit juices collected from in and around the Coimbatore area, TN, India. The findings of this analysis showed that species containing biofilm character and resistant to 6 or more drugs tested.

\section{MATERIALS AND METHODS}

\section{Sample collection}

A total of 6 food samples each comprising 4 numbers; goat, poultry, and fish meat, in case of fruit juices, apple and orange were collected randomly from four different localities of Coimbatore areas. All samples were aseptically collected and then packaged in sterile polythene zip lock bags and carried to the laboratory in aseptic conditions in a cold box within two hours from the time of purchase.

All samples were analyzed within 2-4 hours after their arrival at the laboratory. A sharp sterile knife was used to cut meat samples from surfaces in sterile trays. To isolate bacteria, homogenate meat samples were streaked onto Chromogenic media, MSA media, SS agar media, blood agar media plates and incubated for 18-24 hours at $35+2{ }^{\circ} \mathrm{C}$. In the case of fruit juices, a loop full of samples was directly inoculated into above mentioned media at incubated for $18-24$ hours at $35+2{ }^{\circ} \mathrm{C}$, then genus were confirmed with colony morphology.

\section{Antimicrobial Susceptibility Test}

Susceptibility tests were performed using the Kirby-Bauer method on MuellerHinton agar in accordance with Clinical and Laboratory Standards Institute (CLSI; formerly NCCLS) guidelines (NCCLS 2002) [8] and using 11 antibacterial agents.

\section{Determination of biofilm producing isolates}

The agar medium was prepared by adding $37 \mathrm{~g}$ of the BHI powder, $5 \%$ of sucrose and $10 \mathrm{~g}$ of agar, and Congo red $(0.8 \mathrm{~g} / \mathrm{L})$ in $1 \mathrm{~L}$ of distilled water. The mixture was then autoclaved for $15 \mathrm{~min}$ at $121^{\circ} \mathrm{C}$. Then the media was poured into the petri plates and allowed to solidify. Once 
the media had solidified, the plates were inoculated with the microorganisms and incubated at $37^{\circ} \mathrm{C}$ for $24 \mathrm{~h}$. The plates were observed the next day, the organisms were considered positive (biofilm-producers) when they produced black colonies on the agar and negative (non-biofilm producers) when they produced pink, or red-orange colonies on the Congo red agar [9].

\section{RESULTS AND DISCUSSION}

The distribution of bacterial species per sample is summarized in Figure 1. It showed high contamination levels of the sample with total aerobic bacteria and total and fecal coliforms. The biochemical identification confirmed the presence of $E$. coli, Salmonella spp, E.faecalis, P.aeruginosa, K.pneumoniae, Proteus spp and $S$. aureus.

The prevalence of contamination rate was $79.1 \%$ in meat samples and $21 \%$ in fruit juices samples. Among the meat samples, chicken meat had the highest contamination of 7 bacterial genera $(29.1 \%)$, and $27 \%$ in fish samples. In the case of fruit juices, $12.5 \%$ and $8.3 \%$ were occurrences in apple and orange respectively. E.coli, E.faecalis and K.pneumoniae were the dominant isolates and isolated in this study as they were identified in $21 \%$ of samples, followed by Proteus spp (12.5\%). Shrestha et al. [10] made the same finding in 2017; they were observed the same line of results from chicken meat samples.

During the present analysis, all goat meat samples had different types of bacteria; this level of contamination was significantly lower than in Bhoomika et al [11], which reported $46.34 \%$ of chevon meat samples containing bacterial isolates. The present study summarized that the distribution of the isolated bacterial strains is different from one butcher shop to another shop; likewise, result was observed in fruit juices. Surprisingly S.aureus and Salmonella spp were isolated in limited samples. In 2019 Saud et al [6] also found that the minimum percentage of Salmonella spp and S.aureus from meat samples. Indeed, the statistical study corroborated with this observation and demonstrates that significant difference $(\mathrm{P}>0.05)$ in contamination rates exists between samples with all the tested bacteria.

While animals are given antibiotics for growth promotion or feed utilization output, bacteria are exposed to low doses of these drugs over a long period of time. This is inappropriate antibiotic use and may lead to resistant bacteria being developed. In this present study, all isolates were subjected to the determination of antibiotic susceptibility with 11 antibiotics.

Number of antibiotic resistance patterns and the subsequently a higher number of MDR bacteria were observed in 
goat samples in comparison to other samples. Simultaneously, amongst food isolates, we have no longer found differences in antimicrobial resistance among meats and fruit juices (48.2\% and $43 \%$ respectively). In meat isolates, Proteus sp was high resistance (58\%) and followed by E.faecalis $(53.3 \%)$, in the case of fruit juices isolates, E.coli showed high resistance to antibiotics (Figure 2).

A research conducted in samples of chicken meat in Nepal showed a $44 \%$ prevalence of Proteus sp, of which $100 \%$ isolates were MDR [6]. In disagreement with Gaglio et al. [12], Sanlibaba et al. [13], we detected high resistance to penicillin, amoxicillin and ampicillin. The higher levels of ampicillin resistance in isolates are achieved by the higher levels of penicillinbinding protein 5(PBP5) expressions [14]. This antibiotic is frequently used for the treatment of enterococcal infection [15].
Out of $30 \%$ E.coli isolates from chicken meat samples, $100 \%$ were resistant to Amoxicillin, Penicillin, Cefixime and gentamycin, $67 \%$ to tetracycline, ampicillin and Novabiocin, $33.3 \%$ to Nalidixic acid. Compare to the previous study [6], our result of resistance percentage of Amoxicillin was higher and the same time resistance percentage of Nalidixic acid was low. Out of $30 \%$ K.pneumoniae obtained from chicken raw meat, $67 \%$ were resistant to Nitrofurantoin and Cefixime. The Figure 3 was revealed the prevalence of antibiotic resistance isolates on food samples. From the overall results, Amoxicillin was resistant to $85 \%$ of meat isolates, which was higher than the previous report of Saud et al [6]. Out of 38 isolates, $39.4 \%$ of were MDR isolates, which were highly observed in goat meat samples.

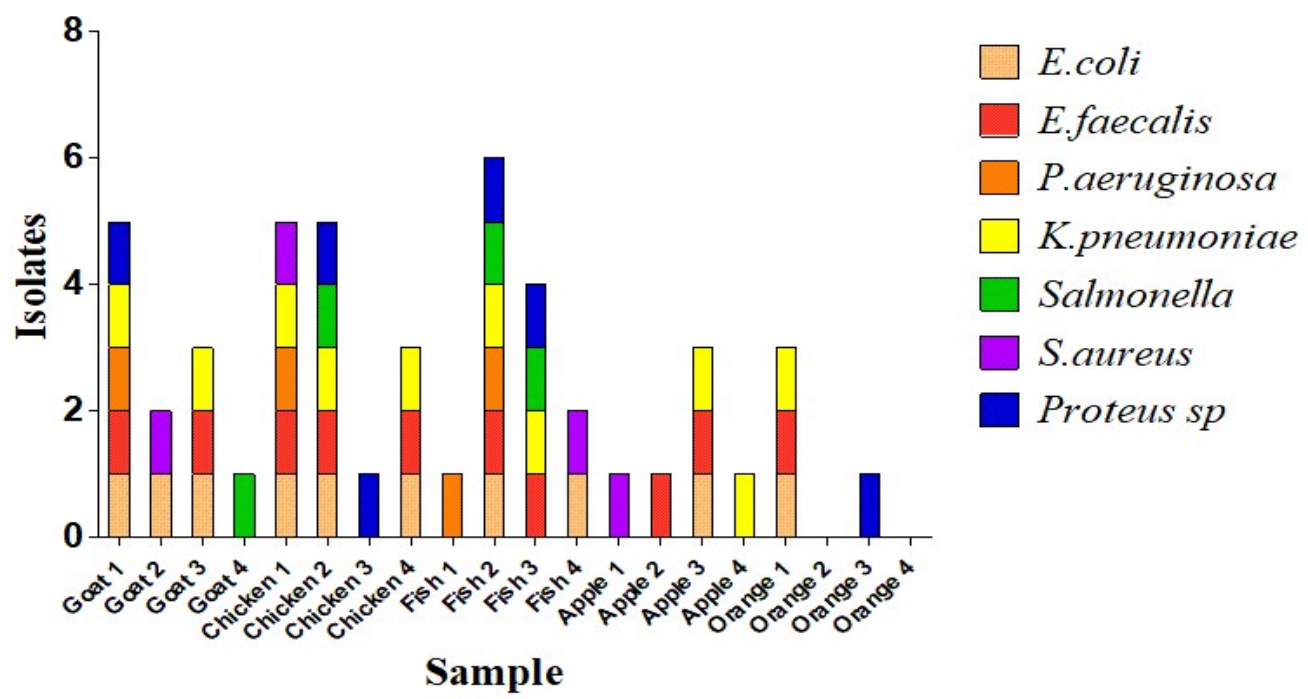

Figure 1: Prevalence of bacterial isolates from food samples 


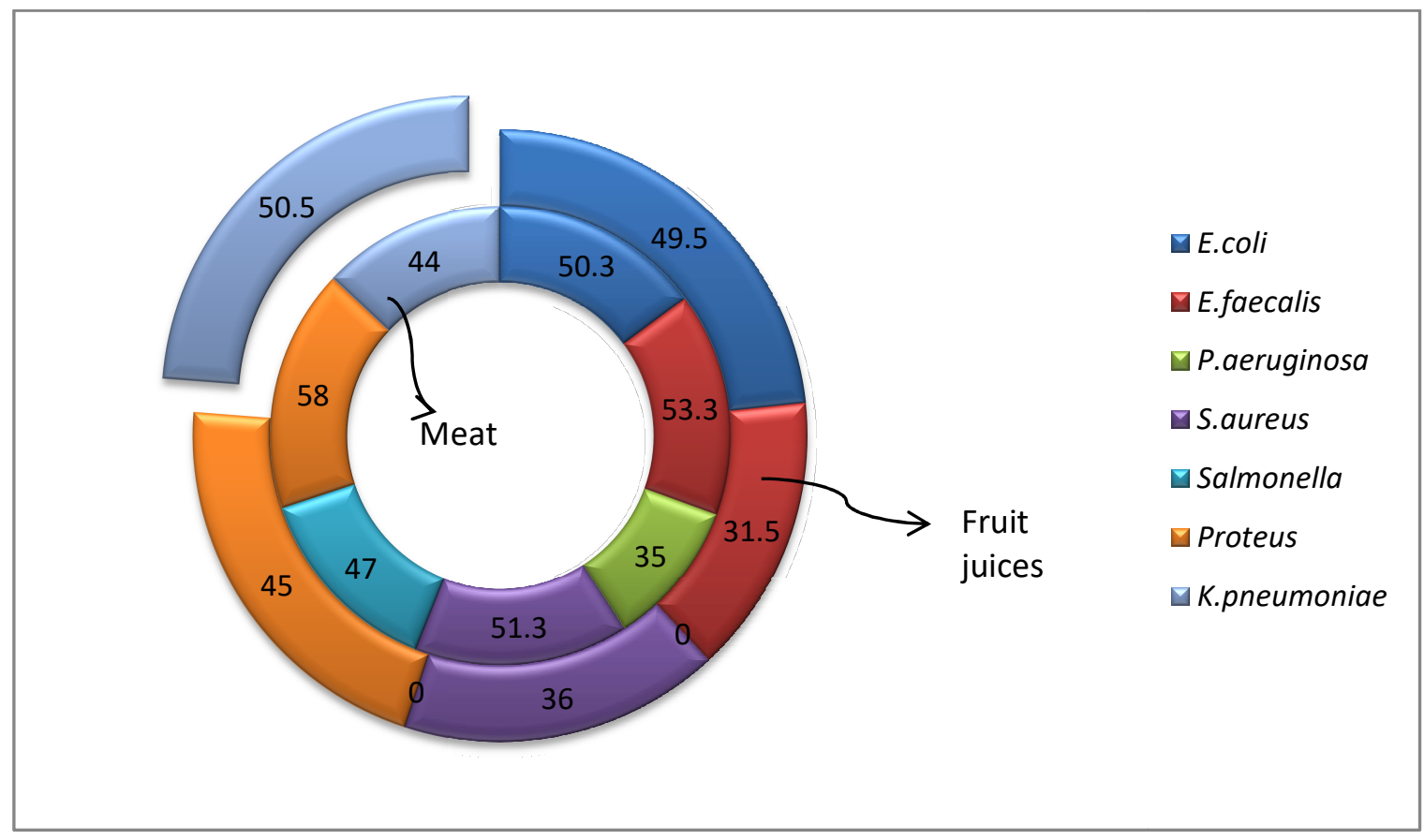

Figure 2: Prevalence of antibiotic resistance on food isolates

*Outer ring denotes the fruit juice samples and inner ring denote the meat samples

\begin{tabular}{|c|c|c|c|c|c|c|c|c|c|c|c|c|c|}
\hline $\begin{array}{l}\text { S. } \\
\text { No }\end{array}$ & ISOLATES & TE & $\mathbf{K}$ & $\mathbf{P}$ & GEN & AMX & $\mathrm{C}$ & NA & AMP & NOR & CFM & NV & $\frac{100 \%}{<05 \%}$ \\
\hline & E.coli & & & & & & & & & & & & $\begin{array}{l}-395 \% \\
<00 \%\end{array}$ \\
\hline & E.faecalis & & & & & & & & & & & & $\leq \mathbf{8 0 \%}$ \\
\hline & Salmonella & & & & & & & & & & & & $\leq 60 \%$ \\
\hline & S.aureus & & & & & & & & & & & & $\leq 40 \%$ \\
\hline & P.aeruginosa & & & & & & & & & & & & $\leq \mathbf{2 0 \%}$ \\
\hline & K.pneumoniae & & & & & & & & & & & & $0 \%$ \\
\hline
\end{tabular}

Figure 3: Percentage of antibiotic resistance on food samples

Following the study of meat, the same effect was observed in fruit juices, and apple and orange juice derived isolates were found to have higher drug resistance, although their size was less than the resistance of the meat-containing isolates. In 2020, Malik et al. [16] were observed the various bacterial isolates from fruit juices. This is a sign of unhealthy conditions, fruit juices are contaminated with unhealthy methods and poor water usage during or after production.

In this study, the antimicrobial efficacy of the fruit juice was found to be higher for a large number of drugs. Among the 10 of fruit juices isolates, 3 of were multidrug resistance and highest drug resistance against to betalactam antibiotics of penicillin and ampicillin. In 2018, Mandal and Mandal [17] was observed the MDR isolates from fruit juices in Malda 
Town, India. Other than India, recently in Bangladesh Sultana et al [18] was found that Amoxicillin resistance isolates of K.pneumoniae from various fruit juices.

From the overall results in both sources, among the 11 antibiotics used, penicillin and amoxicillin were resistant to 93.3\% of isolates and Cefixime resistance to $70 \%$ of isolates. All isolates except K.pneumoniae were $100 \%$ resistant to penicillin and amoxicillin. Presently, most of the first line of antibiotics was resistance to food isolates, especially penicillin and amoxicillin. The observed high rates of resistance may be due to uncontrolled consumption, a consequence of easy access to inefficient and cheap antibiotics. The other reason was due to the production of betalactamase enzyme that hydrolyzes the $\beta$-lactam ring thereby inactivating the drug [19].

Additionally, biofilm producing isolates were determinate in this present study with congo red agar plate method. Biofilm is bacteria engendering extracellular polysaccharide matrix, which plays a consequential role in evading the host immune system and resisting antimicrobial agents, leading to constant and chronic infections. Among the 48 isolates, $29(60.4 \%)$ of were biofilm producers. The highest biofilm producers were observed from goat (38\%) samples and $21 \%$ from chicken and fish meat samples, the lowest percentage $(7 \%)$ of were from orange juices.

Presently, predominant biofilm producers were E.coli and followed by K.pneumoniae. Several researches has been recorded the biofilm producing K.pneumoniae, and E.coli from poultry and goat meat samples $[\mathbf{2 0}, \mathbf{2 1} \&$ 22]. Comparing with non biofilm producers, biofilm producers possess the highest antimicrobial resistance. This phenomenon was a coincidence with earlier studies [23]. In this study betalactam group of antibiotics (penicillin, ampicillin and amoxicillin) and cephalosporins (Cefixime) were highly resistant in biofilm positive isolates. The study by Marques and colleagues [24] was consistent with our report. His study found that biofilm producing isolates were not controlled by betalactam antibiotics.

In poultry and another animal husbandry, some bacterial species are the key causes of infections. Most of the diseases are related to foodborne outbreaks, live contact with animals, poor hygiene and exposure to the environment. The pathogenesis and virulence of these isolates have increased with the advent of antimicrobial resistance, and treatment options are decreased and are highly costly. Multidrug-resistant bacteria have been detected in meat and fruit samples, which endanger both handlers and consumers and a hazard to public health worldwide. The 
above information makes it imperative for the world to monitor antibiotic use in both livestock and humans. The proper antibiotic policy and infection control management in hospital settings is crucial to overcoming the problems associated with infections by MDR strains in humans and animals.

\section{REFERENCE}

[1] Thomas Bintsis. Foodborne pathogens. AIMS Microbiol. 2017; 3(3): 529-563.

[2] Dorothy L Moore. Foodborne infections. Paediatr Child Health. 2008; 13(9): 779-782.

[3] Norma Heredia, Santos García. Animals as sources of food-borne pathogens: A review. Anim Nutr. 2018; 4(3): 250-255.

[4] Karoki WH, Karanja DN, Bebora LC, Njagi LW. Isolation, Characterization, and Quantification of Bacteria from African Sausages Sold in Nairobi County, Kenya. International Journal of Food Science. 2018; 1-9.

[5] Hiroi M., Yamazaki F, Harada T, Takahashi N, Iida N, Noda Y. Prevalence of extended-spectrum Blactamase-producing Escherichia coli and Klebsiella pneumoniae in food-producing animals. $J$. Vet. Med. Sci. 2012; 74: 189-195.

[6] Bhuvan Saud, Govinda Paudel, Sharmila Khichaju, Dipendra
Bajracharya, Gunaraj Dhungana, Mamata Sherpa Awasthi, and Vikram Shrestha. MultidrugResistant Bacteria from Raw Meat of Buffalo and Chicken, Nepal. Veterinary Medicine International. 2019: 1-7.

[7] Matthew O, Chiamaka R, and Chidinma O. Microbial analysis of poultry feeds produced in Songhai farms, Rivers State, Nigeria, Journal of Microbiology \& Experimentation, 2017: 4(2): 1-10.

[8] NCCLS. National Committee for Clinical Laboratory Standards. Performance standards for antimicrobial disk and dilution susceptibility tests for bacteria isolated from animals. Approved standard M31-A2. Wayne, NCCL.

[9] Freeman DJ, Falkiner FR, Keane CT. New method for detecting slime production by coagulase negative Staphylococci. $J$ Clin Pathol, 1989; 42: 872-874.

[10] Shrestha, Anup Muni Bajracharya, Hemraj Subedi, Raju Shah Turha, Sachin Kafe, Saroj Sharma, Sunil Neupane and Dhiraj Kumar Chaudhary. Multi-drug resistance and extended spectrum beta lactamase producing Gram negative bacteria from chicken meat in Bharatpur Metropolitan, 
Nepal. BMC Res Notes, 2017; 10(574): 2-5.

[11] Bhoomika S. Shakya A. Patyal NE. Gade. Occurrence and characteristics of extendedspectrum ß-lactamases producing Escherichia coli in foods of animal origin and human clinical samples in Chhattisgarh, India, Veterinary World, 2016; 9(9); 996-1000.

[12] Gaglio R, Couto N, Marques C, Lopes MFS, Moschetti G., Pompa C, Settani L. Evaluation of Antimicrobial Resistance and Virulence of Enterococci from Equipment Surfaces, Raw Marterials, and Traditional Cheeses. International Journal of Food Microbiology, 2016; 236: 107-114.

[13] Pinar Sanlibaba \& Esra Senturk.Prevalence, characterization and antibiotic resistance of enterococci from traditional cheeses in Turkey. International journal of food properties 2018; 21(1): 19551963.

[14] Vrabec M, Lovayova V, Dudrikova K, Gallo J, Dudrikova E. Antibiotic Resistance and Prevalence of Enterococcus Spp. and Escherichia coli Isolated from Bryndza Cheese. Italian Journal of Animal Science. 2015; 4: 609614.

[15] Frazzon APG, Gama AB, Hermes V, Bierhals CG, Pereira RI, Guedes AG, D'azevedo P, Frazzon J. Prevalence of antimicrobial resistance and molecular characterization of tetracycline resistance mediated by tet( $(\mathrm{M})$ and tet $(\mathrm{L})$ genes in Enterococcus spp. isolated from food in southern Brazil. World J Microbiol Biotechnol. 2010; 26: 365-370.

[16] Malik Y, Omar BJ, Singh A. Bacteriological analysis of streetvended fruit juices available in Rishikesh, Uttarakhand. J Family Med Prim Care, 2020; 9: 938-942.

[17] Sudeshna Mandal and Shyamapada Mandal.Multiple Antibiotic Resistance Indices of Potential Pathogenic Bacteria Isolated from Street Vended Fruit and Sugarcane Juices, Malda Town, India. Acta Scientific Pharmaceutical Sciences. 2018; 2 (10): 89-94.

[18] Sultana F, Marzan LW, Mina SA. Microbiological quality assessment of locally vended and commercially packed fruit juices in Chittagong city of Bangladesh. J. Bio-Sci. 2019; 27: 43-58. 
[19] Warawan Eiamphungporn, Nalini Schaduangrat, Aijaz Ahmad Malik, and Chanin Nantasenamat. Tackling the Antibiotic Resistance Caused by Class A $\beta$-Lactamases through the Use of $\beta$-Lactamase Inhibitory Protein. Int $J$ Mol Sci. 2018; 19 (2222): 1-24.

[20] Suganya, D.Jegadeeshkumar and D. Ravi. Evaluation of Antimicrobial Activity of Solanum Xanthocarpum against

Betalactamase and Biofilm Producing Microorganisms. International journal of novel trends in pharmaceutical sciences. 2014; 4(6): 188-192.

[21] Milanov, Dragana Ljubojevic, Ivana Cabarkapa, N. Karabasil and Maja Velhner. Biofilm as risk factor for Salmonella contamination in various stages of poultry production. Europ. Poult.Sci, 2017; 81: 1-14.

[22] Daehre. ESBL-Producing Klebsiella pneumoniae in the Broiler Production Chain and the First Description of ST3128.
Front. Microbiol. 2018; 9(2302): 1-9.

[23] Zhang, Jun Dong, Yiluo Cheng, Qin Lu, Qingping Luo, Guoyuan Wen, Guoping Liu, "Genotypic diversity, antimicrobial resistance and bioflm-forming abilities of Campylobacter isolated from chicken in Central China. Gut Pathog, 2017; 9(62): 1-10.

[24] Viviane Figueira Marquesa, Cassia Couto da Mottaa, Bianca da Silva, Soaresa, Dayanne Araujo deMeloa, Shana de Mattos de Oliveira, Coelhob, Irene da Silva, Coelhoa, Helene Santos, Barbosaciliane Moreira Soares deSouza. 2017. Biofilm production and beta-lactamic resistance in Brazilian Staphylococcus aureus isolates from bovine mastitis. Brazilian journal of microbiology, 2017; 48: 118124. 